\title{
Advantages of Precession Electron Diffraction in the Study of Small Metallic Nanoparticles
}

\author{
Clemente Fernando ${ }^{1,2}$, M. Mozammel Hoque ${ }^{1}$, José Reyes Gasga ${ }^{2}$ and Arturo Ponce ${ }^{1 *}$ \\ 1. Department of Physics and Astronomy, University of Texas at San Antonio, San Antonio, TX, United \\ States \\ 2. Departamento de Materia Condensada, Instituto de Física, Universidad Nacional Autónoma de \\ México, Circuito de la Investigación Científica s/n, Ciudad Universitaria, Coyoacán, Ciudad de México, \\ México. \\ * Corresponding author: arturo.ponce-pedraza@utsa.edu
}

In the field of nanotechnology defining the atomic structure of a material is of paramount importance in order to understand its properties. In general, the structure of regular solids can be determined via conventional methods like X-ray, neutron, or electron diffraction. In this case, the order in the structure displays long-range characteristics with repeating units that do not vary considerably over the atomic and molecular scale. These long-range conditions are translated in clean, sharp diffraction peaks that allows the retrieval of the structure through the above mentioned conventional crystallographic methods. However, these methods are less efficient to determine the structure of nanomaterials. Structural analysis of crystalline and amorphous materials has been predominantly performed by X-ray diffraction using the pair distribution function (PDF). PDF describes the probability to find two atoms separated a certain distance $r$, providing information on average interatomic distances and coordination. Experimentally, a PDF can be retrieved from X-ray, neutron, or electron diffraction of a powder of identical particles, resulting in highly averaged data but with higher signals compared to the direct intensity diffraction data. Electron diffraction has the advantage to register the patterns at individual nanocrystals. Most of the crystals phases can be inferred by conventional selected area electron diffraction (SAED). However, due to highly dynamical effects caused by the great interaction of electron with matter, it is usually not possible to obtain kinematical diffraction patterns. For this reason, X-rays are still widely used especially for solving crystal structures. In the present work we collected electron diffraction patterns with an axial CMOS (16 bit) camera under precession electron conditions. The patterns have registered and post-processed with ePDF software and SUePDF [1-2]. Quantification with theoretical models have been performed with Diffpy for fiting, using python libraries [3]. Figure 1 shows gold nanoparticles deposited on amorphous carbon films.

In addition, we have analysed by precession electron diffraction the symmetry of metallic nanoparticles. Symmetry of non-translational crystalline structures such as metallic and semiconductor nanoparticles is normally determined by the Bravais lattices. At nanoscale, shape and size of particles play an essential role in their physicochemical properties such as optical, catalytical, electronic and magnetic properties. For instance, noble metals exhibit different surface plasmon resonances depending on the size and shape of metallic nanoparticles. Particularly, the magneto-optical interactions have gained attention due to the potential technological applications in spintronic, electromagnetic shielding, magneto-optical data storage and others. Nanoscale magnetic structures, and their ordered arrays, are being considered for use in several advanced technological areas such as MEMS and power devices as supercapacitors or batteries. In this work we have performed a systematic analysis of gold decahedra nanoparticles ranging from $10 \mathrm{~nm}$ up to $300 \mathrm{~nm}$. The analysis was carried out using nano-beam electron diffraction on the nanoparticles oriented along the fivefold symmetry, in which five entities are identified. In order to 
increase the number of reflections and to obtain quasi-kinematical diffraction patterns we have applied precession electron diffraction in the analysis. The electron diffraction patterns oriented in the fivefold show the reflections of the five crystals separated by the multiple twins. Centrosymmetric patterns are observed in nanoparticles below $70 \mathrm{~nm}$; however bigger nanoparticles present a symmetry loss in the diffraction patterns. The non-centrosymmetric patterns were found by connecting the reflections $+g$ and -g of all the 111 reflections. In this presentation we discuss the reason of the symmetry loss and how the precession angle affects the analysis. In this work we used the nano-beam electron diffraction with a probe size of $1.5 \mathrm{~nm}$ on a decahedral gold nanoparticle along.

\section{References:}

[1] J Shanmugama et al., SoftwareX 6 (2017), p. 185.

[2] DT Tran et al., J. Appl. Cryst. 50 (2017), p. 304.

[3] P Juhás et al., J. Appl. Crystallogr. 46 (2013), p. 560.
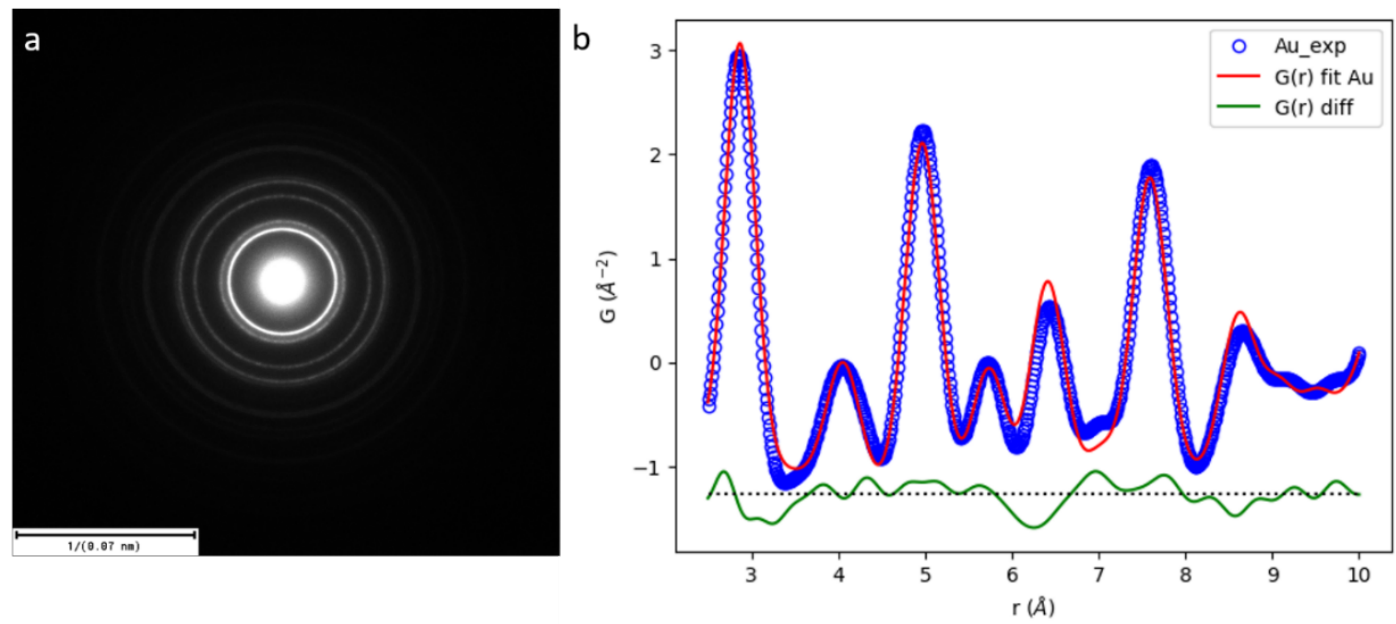

Figure 1. Figure 1. a) Experimental electron diffraction pattern of gold film. b) Experimental electron PDF (blue) compared to PDF for ideal FCC gold (red). The residual difference between the two graphs (green) was calculated as $15 \%$.

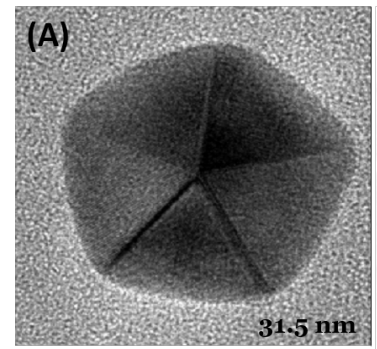

(B)

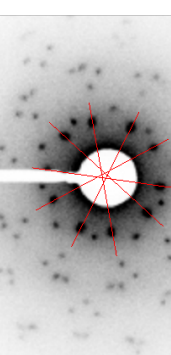

(C)

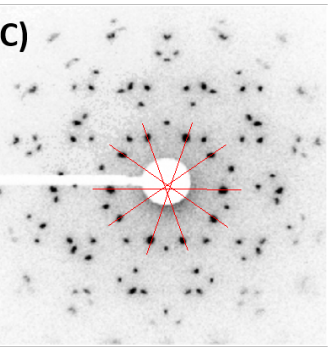

Figure 2. a) high resolution transmission electron microscopy image, b) nanobeam electron diffraction patterns b) with and c) without precession electron diffraction. 